\title{
ANÁLISIS DE LA PRODUCCIÓN CIENTÍFICA ENFERMERA SOBRE EMPODERAMIENTO FEMENINO Y SALUD PERINATAL ${ }^{1}$
}

\author{
María del Carmen Martín-Bellido'; Juan Diego González-Sanz². \\ Correo electrónico: mamenmb22@gmail.com
}

1. Graduada en Enfermería. Residencia de Ancianos Dr. Bartolomé García GonzálezToruño. La Zarza-Perrunal, Huelva.

2. Doctor en Ciencias Sociales Aplicadas (Filosofía), Enfermero especialista en Obstetricia y Ginecología (Matrona). Departamento de Enfermería, Universidad de Huelva.

Recibido: 30/05/2019 Aceptado: 08/11/2019

${ }^{1}$ Una versión preliminar de este trabajo fue presentada como comunicación oral en la IV Reunión Internacional de Investigación y Educación Superior en Enfermería celebrada en Granada entre el 8 y el 9 de noviembre de 2018, con el título: «¿Qué producen las enfermeras sobre empoderamiento femenino en el cuidado perinatal?». Posteriormente, una versión ampliada fue presentada como Trabajo Fin de Máster del Máster de Investigación en Ciencias Sociosanitarias de la Universidad de León en 2019. 


\section{RESUMEN}

\section{Introducción}

El concepto de empoderamiento femenino se utiliza con una frecuencia cada vez mayor en el campo de la enfermería, concretamente por las matronas, apareciendo especialmente en el ámbito de la salud perinatal de la mano de ideas como la humanización del parto o la prevención de la violencia.

\section{Objetivos}

Analizar la producción científica sobre empoderamiento femenino en el ámbito perinatal.

\section{Metodología:}

Estudio bibliométrico de la producción científica en las revistas Birth, Women and Birth y Midwifery. El análisis de datos se llevó a cabo a partir de su clasificación y categorización. Además, se definieron conceptualmente, enunciaron y codificaron las categorías.

\section{Resultados y discusión}

Se identificaron un total de 341 artículos, de los cuales 44 se seleccionaron por su relevancia. Sin embargo, solo 13 cumplían con los criterios de inclusión del estudio. A pesar de que los artículos seleccionados tenían diferentes objetivos y analizaban el empoderamiento femenino desde distintas perspectivas, fue posible realizar un acercamiento gracias a la clasificación y categorización del contenido de los mismos. Parece ser que son aspectos claves del empoderamiento femenino: la confianza entre la matrona y los pacientes; sentirse escuchada por los profesionales de la salud; aceptar el dolor; el soporte físico y psicológico por personas cercanas o profesionales; los cuidados personalizados; la cercanía y la empatía de los profesionales; la privacidad y la comunicación como elementos clave.

\section{Conclusiones}

El empoderamiento femenino en el ámbito de la salud perinatal no es un tema tratado con amplitud en las revistas estudiadas, ya que no existe una definición unívoca del mismo. Sin embargo, podemos establecer como aspectos clave del empoderamiento femenino la confianza entre la matrona y las gestantes, la escucha activa, la aceptación del dolor, la presencia de soporte físico y mental, la personalización de los cuidados, la empatía y cercanía de los profesionales, la privacidad, y la comunicación como elemento clave empoderador.

Palabras clave: empoderamiento femenino; cuidados perinatales; enfermería; producción científica. 


\section{ABSTRACT}

\section{Introduction}

The concept of female empowerment is often used in nursing by midwifes, since it has become a key topic and discussion for said profession. Accordingly, it is used in perinatal health hand in hand with ideas such as humanized childbirth and the prevention of violence.

\section{Objectives}

The aim of this study is to analyze the scientific production on female empowerment and perinatal health.

\section{Methodology}

A bibliometric study of the scientific production published in the journals Birth, Women and Birth and Midwifery was carried out. The analysis was realized through the classification and categorization of the data. Moreover, the categories were specified, encoded and conceptually defined.

\section{Results and Discussion}

341 articles were identified, and 44 were selected because of their relevance. However, only 13 matched the inclusion criteria. Given that selected articles have different aims and analyze the notion of empowerment differently, it was only possible to discuss the topic through their classification and categorization. Accordingly, the most relevant aspects concerning the sense of female empowerment arising out of the interaction between patients and midwifes are: feeling that health professionals listen to what you have to say; accepting pain; the physical and psychological support of close people or professionals; personalized care; health professionals' empathy and closeness; privacy; and the role communication as key element for empowerment.

\section{Conclusions}

Female empowerment in the perinatal period is an understudied discussed topic in the journals analyzed in this article. That is the reason why there is not a univocal definition of the term. It could be argued that female empowerment is much dependent on aspects such as confidence between patients and midwife, the feeling of being listened by health professionals; the acceptance of pain; the physical and psychological support of close people or professionals; personalized care; health professionals' empathy and closeness; privacy; and a fluid communication.

Keywords: Empowerment; Perinatal Care; Nursing; Scientific Production. 


\section{INTRODUCCIÓN}

El concepto de empoderamiento femenino se está utilizando con una frecuencia creciente en el ámbito de las ciencias de la salud y especialmente en el campo de la enfermería. La Organización Mundial de la Salud (2001) define el empoderamiento como «un proceso continuo a través del cual individuos y comunidades adquieren confianza, autoestima, conocimientos y poder necesarios para satisfacer las necesidades esenciales y para tomar el control de sus vidas, específicamente en el ámbito de la salud».

Por otra parte, Mouta Oliveira et al. (2017) lo entienden como «una forma de ganar poder interior y tener el control sobre todo aquello que te está sucediendo, así como la defensa de los propios derechos». A su vez, Cardoso Da Silva et al. (2016) indican que es un «proceso a través del cual se pretende promover y mejorar la habilidad individual de satisfacción de las propias necesidades», lo que aporta sensación de control tanto a las vidas de los individuos como a la de la comunidad. Camberos Sánchez (2011) añade a lo anterior que «el empoderamiento femenino modifica la imagen de las mujeres sobre sí mismas y las creencias sobre sus derechos y capacidades».

Hay pocas áreas en la vida de las mujeres donde se ponga más en riesgo su autoimagen y sus creencias que el ámbito de la salud sexual y reproductiva. En este campo, numerosos autores afirman que el empoderamiento femenino permite a la mujer entender su cuerpo y potenciar la capacidad de dar a luz, así como sus emociones. Kwee y McBride (2015) señalan en esta línea que «la intensidad de la vivencia del parto parece estar directamente relacionada con el empoderamiento». De este modo, empoderar a las madres puede considerarse una iniciativa beneficiosa si tenemos en cuenta que, como afirma Coral Ibarra (2008), la mujer embarazada sufre una especial vulnerabilidad emocional y social. Se trata, una vez más, de introducir la humanización en el proceso de parto, definida según González Sanz et al. (2012) como «la adquisición y puesta en práctica por parte de los profesionales sanitarios de un conjunto de conocimientos, habilidades y actitudes que configuran una forma diferente de llevar a cabo su labor asistencial, [...] abandonando el modelo medicalizado clásico para incorporar un nuevo modelo que pone énfasis en el uso de prácticas estrictamente necesarias [...], lo que supone el desarrollo de la autonomía de la mujer».

Una de las muchas vías a través de las que se está intentando desarrollar esta iniciativa es el ofrecimiento del plan de parto a las madres gestantes. Dicho plan es un documento 
que ayuda a la embarazada a clarificar y dejar constancia de sus propios deseos, expectativas y necesidades durante el parto. Se busca con este plan que la mujer tome el control de su situación, exprese sus deseos y se facilite la toma de decisiones informadas, además de favorecer el cumplimiento de sus derechos (Biurrun-Garrido y Goberna Tricas, 2013). Por otra parte, el uso del plan de parto también puede favorecer el aumento de la calidad de los cuidados, así como la individualización de la atención. Sin embargo, para que esto sea así es fundamental que, como indican Mouta Olivera et al. (2017), los planes de parto se den a conocer a la población y estén accesibles tanto en el periodo de gestación como al llegar al centro donde tendrá lugar el parto.

Ya sea a través de la oferta, explicación y/o ejecución del plan de parto, o de otras actividades, parece claro que unos de los colectivos profesionales más implicados en este asunto son las enfermeras y, más concretamente, las matronas. Sánchez-Jiménez et al. (2007) han destacado la capacidad de la matrona para fomentar la participación de la población en la formación sobre autocuidados. Esto se debe al contacto directo entre los pacientes y estas profesionales, que son capaces de captar e informar adecuadamente a la población más vulnerable.

De esta forma, estamos de acuerdo con Mouta Oliveira et al. (2017) cuando afirman que «el empoderamiento femenino en el ámbito perinatal puede ser considerado un movimiento enfermero educativo, cuyo objetivo es aportar conocimientos, actitudes y habilidades a las embarazadas para que asuman el poder y la responsabilidad de las decisiones que toman». En este sentido, las matronas pueden jugar el papel de «mecanismo facilitador de la adaptación a las nuevas situaciones de salud» (Cardoso Da Silva et al., 2016).

No obstante, hay que resaltar que este enfoque centrado en los profesionales no es suficiente. Aunque según Mouta Oliveira et al. (2017) «esta posición favorece que las enfermeras puedan garantizar unas actuaciones basadas en el respeto sin la utilización de intervenciones innecesarias reconociendo los aspectos culturales y sociales que influyen en el proceso del parto»), hay que tener en cuenta la objeción de Goberna Tricas y Giménez Segura (2013) insitiendo en los aspectos sociales y culturales como fundamentales cuando afirman «que la atención no tecnificada y respetuosa con la fisiología no garantizará el empoderamiento de la mujer, ya que también influyen los aspectos sociales y culturales». 
Quedando bien establecido, de este modo, que el empoderamiento femenino en el ámbito perinatal es un campo de trabajo más que notable para la enfermería, parece oportuno preguntarse acerca del modo en que está siendo tratado como objeto de investigación. Siguiendo a Luque Ortega et al. (2010), sostenemos que «la producción científica es un buen indicador del estado de desarrollo de la enfermería como ciencia y de su capacidad de generar nuevos conocimientos». Por ello se nos hace indispensable la realización de estudios bibliométricos qué evalúen la presencia de investigaciones sobre este tema en las revistas científicas de enfermería. La observación atenta de estos trabajos nos permitirá conocer más acerca de la amplitud y la calidad de la producción científica enfermera sobre esta cuestión.

Y es que, según afirma Reina Leal et al. (2013), «el análisis de las referencias a través del estudio bibliométrico permite conocer el desarrollo de los procesos de comunicación científica en las disciplinas», por lo que «se ha convertido en un elemento clave para los agentes evaluadores de la actividad científica, quienes se apoyan en los indicadores bibliométricos para clasificar revistas» (Reina Leal et al., 2013).

De acuerdo con los estándares actuales de medición de la calidad de las publicaciones científicas, se considera como de mayor relevancia a aquellas revistas incluidas en bases de datos de citas como el Journal Citation Reports (JCR). La mayoría de estas revistas provienen del ámbito anglosajón y publican sus contenidos en inglés. Por ello, y dadas nuestras limitaciones para un estudio más amplio, nos ceñiremos, en este estudio, a las revistas incluidas en el primer cuartil del JCR, y dentro de estas a las de mayor nivel de impacto.

\section{OBJETIVOS}

El objetivo general de este estudio es analizar la producción científica de enfermería sobre empoderamiento femenino en el ámbito perinatal. Además, como objetivo específico, nos hemos planteado describir qué significados adquiere la palabra «empoderamiento» en la bibliografía consultada.

\section{METODOLOGÍA}

Se presenta un estudio bibliométrico de la producción científica enfermera sobre el tema de estudio, realizado a través de los análisis de los artículos publicados en revistas de alto 
impacto (incluidas en el Journal Citation Reports de Clarivate Analytics, concretamente en el primer cuartil). Las revistas seleccionadas fueron Birth, Women and Birth y Midwifery, ya que, dentro de la amplia gama de revistas que realizan estudios relacionados con el cuidado perinatal, son las que mayor impacto presentan dentro de la clasificación JCR. El motivo que llevó a limitar nuestro corpus a estas tres revistas fue el volumen de los datos a manejar.

\section{Estrategia de búsqueda}

En primer lugar, se consultó la página del DECS y MESH para conocer los tesauros especializados para la realización de la búsqueda de artículos. Se utilizaron tanto los términos del lenguaje natural como los de lenguaje controlado.

La base de datos consultada fue la Web of Science (WOS). La estrategia de búsqueda fue («Empowerment AND perinatal care»).

\section{Criterios de inclusión}

Se incluyeron artículos originales que cumplieran los siguientes criterios de inclusión: a) estudios que incluían en su título o resumen la palabra «empowerment»o algún término que hiciera referencia a ella (como «confidence» o «birth experience»); b) artículos que permitieran el acceso al texto completo de forma gratuita o al que pudiéramos acceder con las credenciales de la Universidad de Huelva, de la Universidad de León o de la Fundación Index de Enfermería. No se estableció ningún límite temporal en la selección de artículos.

\section{Procedimientos y extracción de datos}

En primer lugar, la selección de artículos se realizó en base al título y resumen. Posteriormente, se accedió al texto completo de los artículos considerados relevantes. Este proceso de recolección de datos fue realizado desde junio hasta octubre de 2018. 


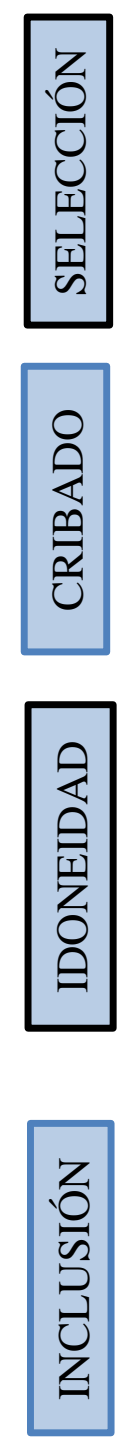

Artículos identificados en las búsquedas en las bases de datos (341)

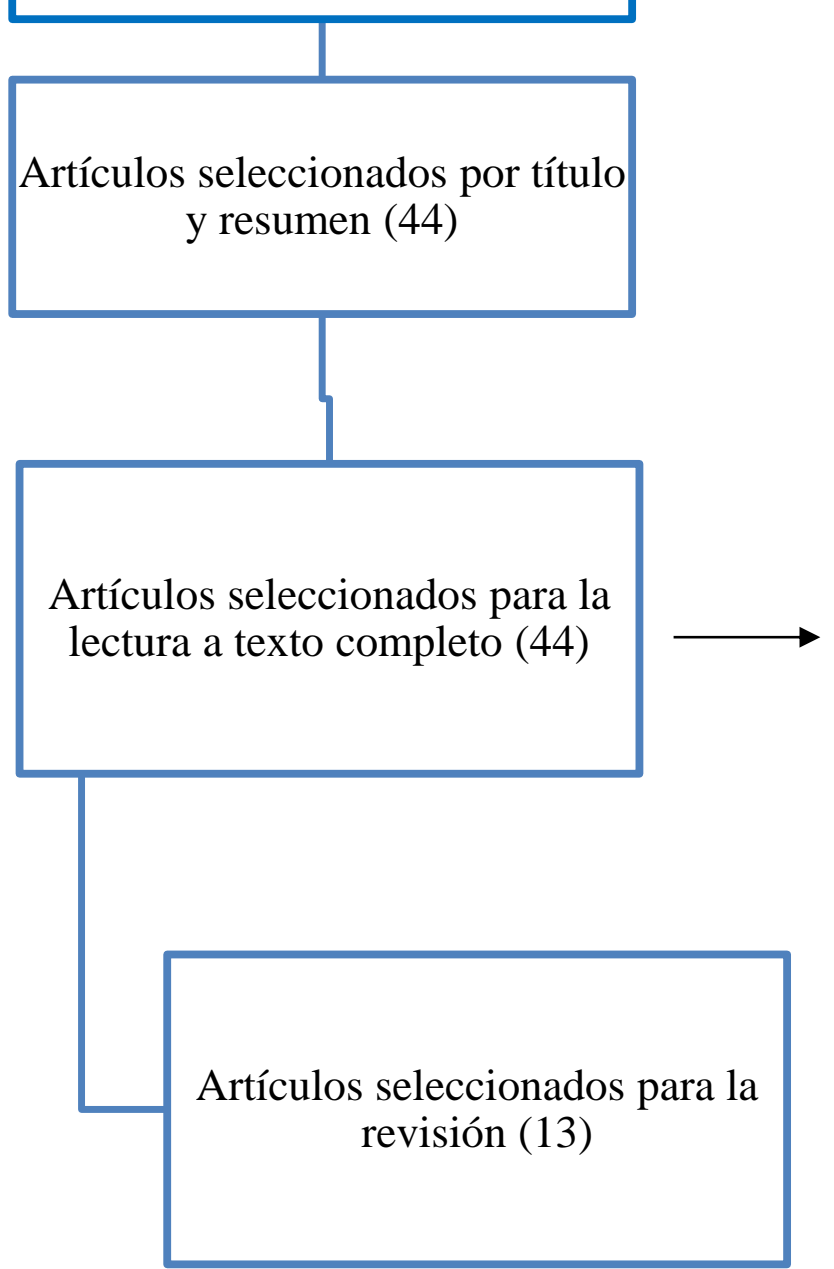

Artículos excluidos (no cumplen criterio inclusión) (31)

Figura 1. Flujograma del proceso de selección de artículos

Tras la selección de los estudios que cumplían los criterios de inclusión, se procedió a la lectura exhaustiva de todos los artículos. Se tuvieron en cuenta aquellos términos considerados como sinónimos de «empoderamiento» o que hacían alusión al empoderamiento.

El análisis de los datos extraídos se llevó a cabo a través de su clasificación, categorización y codificación, extrayendo la máxima información. Se agruparon los términos en función de su similitud y, finalmente, se eligió un término que encabezase la categoría en el cual se pudieran incluir el resto de los términos de esa categoría. También se establecieron subcategorías.

Una vez realizada la categorización, se procedió a la asignación de un código a cada categoría para facilitar el análisis de los datos. Estas categorías fueron definidas 
conceptualmente, enunciadas y codificadas. Toda esta información fue transcrita al programa Word, donde se elaboró una tabla con distintas categorías (Tablas 2, 3 y 4).

Para finalizar, se definieron las conclusiones de cada una de las categorías, agrupándolas según temáticas, y se establecieron los significados que otorga cada revista estudiada al término «empoderamiento».

La definición de los términos relacionados con el empoderamiento utilizados para la categorización de los datos fueron los siguientes:

- Confianza en sí misma: la mujer percibe un aumento de la seguridad sobre sí misma.

- Confianza en el otro: la mujer percibe un aumento de la sensación de seguridad respecto al profesional que la atiende.

- Sensación de control: la mujer percibe una mayor capacidad de decisión sobre la situación en la que se encuentra.

- Aceptación: la mujer muestra conformidad con lo que le está ocurriendo.

- Capacidades: desarrollo de habilidades y destrezas personales y técnicas.

- Soporte tácito: la mujer percibe un aumento del apoyo emocional.

- Soporte táctil: la mujer percibe demostraciones de apoyo físico.

- Control: la mujer percibe un determinado nivel de autoridad sobre el funcionamiento del proceso.

- Humanización de cuidados: la mujer percibe actitudes más amables y humanas durante el proceso.

- Entorno: espacio agradable en lugar y acompañamiento percibido por la mujer en el proceso perinatal.

\section{RESULTADOS}

Como indica el flujograma anterior (figura 1), 13 artículos fueron seleccionados por siguiendo los criterios de inclusión del estudio. En la tabla 1 puede verse el origen de cada uno de los artículos seleccionados. 
Tabla 1. Relación entre artículos seleccionados y las revistas de procedencia

\begin{tabular}{|c|c|c|c|c|}
\hline Revista & $\begin{array}{c}\text { Artículos } \\
\text { hallados en la } \\
\text { búsqueda }\end{array}$ & $\begin{array}{c}\text { Artículos } \\
\text { relacionados } \\
\text { con la temática }\end{array}$ & $\begin{array}{c}\text { Artículos } \\
\text { seleccionados }\end{array}$ & \multirow{2}{*}{ Tesauros } \\
\hline BIRTH & 75 & 20 & 5 & \multirow{2}{*}{$\begin{array}{c}\text { Empowerment» } \\
\text { AND «perinatal } \\
\text { care» }\end{array}$} \\
\hline $\begin{array}{c}\text { WOMEN AND } \\
\text { BIRTH }\end{array}$ & 63 & 10 & 1 & 7 \\
\hline MIDWIFERY & 203 & 14 & & 5 \\
\hline
\end{tabular}

La revista Birth es la que posee el mayor índice de impacto de entre las consultadas $(2.329 ; 2018)$. A pesar de encontrar 75 artículos relacionados con el tema, solo 5 cumplían los criterios de inclusión. En la revista Women and Birth, también aparecen 10 estudios relacionados con el tema, de los que solo hemos podido acceder a 1 de ellos. En el caso de Midwifery, se han seleccionados 14 artículos por contener información relacionada con nuestro estudio. Sin embargo, solo 7 estaban disponibles en la modalidad gratuita.

En cuanto a los resultados obtenidos sobre el significado de «empoderamiento», en la tabla 2 se expresan los términos englobados bajo el concepto de empoderamiento en los artículos analizados procedentes de la revista Birth. Es preciso destacar que 3 de los 5 artículos consultados pertenecientes a esta revista señalan como una cuestión importante dentro del empoderamiento femenino la confianza que se establece entre la matrona y la paciente. Otro tema considerado de relevancia por 2 de los 5 artículos es la aplicación de cuidados individualizados.

Por otro lado, la tabla 3 muestra los términos englobados bajo el concepto de empoderamiento femenino durante el periodo perinatal de la revista Women and Birth. En esta revista también se hace alusión a la confianza que deposita la paciente en los profesionales que la atienden durante el periodo perinatal.

Finalmente, en la tabla 4 se pueden apreciar los resultados de los términos que hacen referencia indirectamente al empoderamiento femenino en el ámbito perinatal en los artículos procedentes de la revista Midwifery. Como aspectos a destacar, se encuentra la relación entre la matrona y la mujer, resaltado por 5 de los 7 artículos analizados. Asimismo, cabe resaltar la importancia de la continuidad de los cuidados y del soporte tanto físico como emocional y espiritual en 3 de los 7 estudios seleccionados. 


\begin{tabular}{|c|c|c|c|c|c|c|c|c|}
\hline \multicolumn{2}{|c|}{ CONFIANZA } & \multirow[b]{2}{*}{$\begin{array}{l}\text { SENSACION DE } \\
\text { CONTROL }\end{array}$} & \multirow[b]{2}{*}{ ACEPTACIÓN } & \multirow[b]{2}{*}{ CAPACIDADES } & \multicolumn{2}{|c|}{ SOPORTE } & \multirow{2}{*}{$\begin{array}{c}\text { HUMANIZACIÓN } \\
\text { DE LOS } \\
\text { CUIDADOS }\end{array}$} & \multirow[b]{2}{*}{ ENTORNO } \\
\hline EN SÍ MISMA & EN OTROS & & & & TÁCITO & TÁCTIL & & \\
\hline $\begin{array}{l}\text { Confianza en sus } \\
\text { habilidades para } \\
\text { identificar sus } \\
\text { propias } \\
\text { necesidades (1) } \\
\text { Ausencia de } \\
\text { preocupaciones } \\
\text { (1) } \\
\text { Aumento de la } \\
\text { autoestima (2) }\end{array}$ & $\begin{array}{l}\text { Confianza en la } \\
\text { matrona y su } \\
\text { responsabilidad } \\
(1,3,4) \\
\text { Confianza en la } \\
\text { situación (1) }\end{array}$ & $\begin{array}{l}\text { Sensación de estar } \\
\text { guiada por la matrona } \\
\text { (1) } \\
\text { Tener armonía con } \\
\text { ellas mismas (1) } \\
\text { Sentirse escuchadas (1) } \\
\text { Sentir que tienen } \\
\text { capacidad de elección } \\
\text { (5) } \\
\text { Manejo de situaciones } \\
\text { (1) } \\
\text { Aceptación de la } \\
\text { situación (3) } \\
\text { Ausencia de miedo (1) } \\
\text { Tener conocimientos } \\
\text { útiles (4) }\end{array}$ & $\begin{array}{l}\text { Ayuda a aceptar } \\
\text { el dolor (1) }\end{array}$ & $\begin{array}{l}\text { Elección de la } \\
\text { persona que la } \\
\text { acompaña. (1) } \\
\text { De la matrona para } \\
\text { hacerle creer a la } \\
\text { mujer que puede. } \\
\text { (1) } \\
\text { Identificar sus } \\
\text { propias } \\
\text { necesidades. (1) } \\
\text { Comunicación (4, } \\
\text { 5) }\end{array}$ & $\begin{array}{l}\text { Palabras y } \\
\text { acciones } \\
\text { específicas (1) } \\
\text { Atención no } \\
\text { verbal (1) } \\
\text { Soporte mental } \\
\text { de la pareja o de } \\
\text { personas } \\
\text { cercanas (1) } \\
\text { Comunicación, } \\
\text { tiempo para } \\
\text { hablar (3). }\end{array}$ & $\begin{array}{l}\text { Físico (1, } \\
2)\end{array}$ & $\begin{array}{l}\text { Buena actitud del } \\
\text { personal sanitario } \\
\text { (2) } \\
\text { Relación con la } \\
\text { matrona (1) } \\
\text { Sensibilidad (3) } \\
\text { Empatía (3). } \\
\text { Cuidados } \\
\text { personalizados/ } \\
\text { individualizados (3, } \\
\text { 5) } \\
\text { Cercanía (3) } \\
\text { Benevolencia (3) } \\
\text { Información } \\
\text { objetiva y clara (3). }\end{array}$ & $\begin{array}{l}\text { Ambiente } \\
\text { cálido (1) } \\
\text { Silencio (1) } \\
\text { Actos } \\
\text { insignificantes } \\
\text { pueden ser } \\
\text { expresados } \\
\text { como } \\
\text { estresantes (1) }\end{array}$ \\
\hline
\end{tabular}

1. Linfgren y Erlandsson (2010); 2. Penny (1991); .3. Lundqvist et al. (2002); 4. Riggs et al. (2016); 5. Eilen et al. (2007) 


\begin{tabular}{|c|c|c|c|c|c|c|c|c|}
\hline \multicolumn{2}{|c|}{ CONFIANZA } & \multirow{2}{*}{$\begin{array}{c}\text { SENSACIÓN } \\
\text { DE CONTROL }\end{array}$} & \multirow[b]{2}{*}{ ACEPTACIÓN } & \multirow[b]{2}{*}{ CAPACIDADES } & \multicolumn{2}{|c|}{ SOPORTE } & \multirow{2}{*}{$\begin{array}{c}\text { HUMANIZACIÓN DE } \\
\text { LOS CUIDADOS }\end{array}$} & \multirow[b]{2}{*}{ ENTORNO } \\
\hline $\begin{array}{c}\text { EN SÍ } \\
\text { MISMA }\end{array}$ & $\begin{array}{c}\text { EN } \\
\text { OTROS }\end{array}$ & & & & TÁCITO & TÁCTIL & & \\
\hline $\begin{array}{l}\text { Tener } \\
\text { conocimientos } \\
\text { sobre el tema } \\
\text { (1) }\end{array}$ & $\begin{array}{l}\text { Confianza } \\
\text { en la } \\
\text { matrona (1) }\end{array}$ & $\begin{array}{l}\text { Colaboración con } \\
\text { el personal } \\
\text { sanitario (1) } \\
\text { Sentirse } \\
\text { escuchada (1) } \\
\text { Recibir } \\
\text { información de lo } \\
\text { que está pasando } \\
\text { (1) } \\
\text { Sentirse parte } \\
\text { integral del } \\
\text { equipo (1) } \\
\text { Participación (1) }\end{array}$ & & $\begin{array}{l}\text { Comunicación y } \\
\text { actitud del } \\
\text { personal sanitario } \\
\text { (1) } \\
\text { Responsabilidad } \\
\text { (1) } \\
\text { Respeto hacia las } \\
\text { decisiones de la } \\
\text { mujer (1) }\end{array}$ & $\begin{array}{l}\text { Guía por } \\
\text { parte de la } \\
\text { matrona y } \\
\text { soporte (1) }\end{array}$ & $\begin{array}{l}\text { Presencia } \\
\text { física de la } \\
\text { matrona (1) } \\
\text { Contacto } \\
\text { físico (1) }\end{array}$ & $\begin{array}{l}\text { Interacción entre la } \\
\text { matrona y la mujer (1) } \\
\text { Comunicar sus deseos } \\
\text { (1) } \\
\text { Tomar decisiones con el } \\
\text { consentimiento de la } \\
\text { mujer, juntos y en } \\
\text { acuerdo. (1) } \\
\text { Trabajo en equipo (1) }\end{array}$ & \\
\hline
\end{tabular}

1. Jödin et al. (2018). 
Tabla 4. Términos de los artículos pertenecientes a la revista Midwifery sobre empoderamiento

\begin{tabular}{|c|c|c|c|c|c|c|c|c|}
\hline \multicolumn{2}{|c|}{ CONFIANZA } & \multirow{2}{*}{$\begin{array}{c}\text { SENSACIÓN } \\
\text { DE CONTROL }\end{array}$} & \multirow[b]{2}{*}{ ACEPTACIÓN } & \multirow[b]{2}{*}{ CAPACIDADES } & \multicolumn{2}{|c|}{ SOPORTE } & \multirow{2}{*}{$\begin{array}{c}\text { HUMANIZACIÓN DE } \\
\text { LOS CUIDADOS }\end{array}$} & \multirow[b]{2}{*}{ ENTORNO } \\
\hline $\begin{array}{c}\text { EN SÍ } \\
\text { MISMA }\end{array}$ & EN OTROS & & & & TÁCITO & TÁCTIL & & \\
\hline $\begin{array}{l}\text { Tomar } \\
\text { decisiones } \\
\text { por ella } \\
\text { misma (1) } \\
\text { Sensación de } \\
\text { crecimiento } \\
\text { personal (2) }\end{array}$ & $\begin{array}{l}\text { Relación } \\
\text { entre la } \\
\text { matrona y la } \\
\text { mujer }(1,2,3 \\
4,5) \\
\text { Cercanía de } \\
\text { la matrona } \\
(3) \\
\text { Respeto de } \\
\text { los valores y } \\
\text { creencias de } \\
\text { la mujer (1) }\end{array}$ & $\begin{array}{l}\text { Sentirse segura } \\
\text { (3) } \\
\text { Alivio del dolor } \\
\text { (3) } \\
\text { Sentirse protegida } \\
\text { (3) } \\
\text { Sensación de } \\
\text { comodidad (3) } \\
\text { Sentirse } \\
\text { escuchada (1 } \\
\text { Sentirse parte del } \\
\text { proceso (4, 5) } \\
\text { Sentirse especial } \\
\text { (3) } \\
\text { Interacción con la } \\
\text { matrona (3) }\end{array}$ & $\begin{array}{l}\text { Hablar } \\
\text { positivamente } \\
\text { del parto (5) } \\
\text { Ayuda para } \\
\text { aceptar el dolor } \\
\text { (5) }\end{array}$ & $\begin{array}{l}\text { Comunicación (1, } \\
\text { 3) } \\
\text { Posibilitar a la } \\
\text { mujer la toma de } \\
\text { decisiones } \\
\text { informadas (3) }\end{array}$ & $\begin{array}{l}\text { Soporte } \\
\text { emocional } \\
(5) \\
\text { Soporte } \\
\text { físico, } \\
\text { emocional } \\
\text { y espiritual } \\
(1,2,4,5)\end{array}$ & $\begin{array}{l}\text { Presencia } \\
\text { de la } \\
\text { matrona } \\
\text { (1) }\end{array}$ & $\begin{array}{l}\text { Cercanía de la matrona (2) } \\
\text { Cuidados personalizados } \\
(1,3,5,6) \\
\text { Empatía (2) } \\
\text { Trabajo en equipo (2) } \\
\text { Continuidad de cuidados } \\
(1,2,3,5) \\
\text { Considerar los deseos y } \\
\text { preferencias de la mujer } \\
\text { (3) } \\
\text { Ratio paciente-matrona (1) }\end{array}$ & $\begin{array}{l}\text { Tener a todos } \\
\text { sus seres } \\
\text { queridos cerca } \\
(3,5) \\
\text { Privacidad (3) }\end{array}$ \\
\hline
\end{tabular}

1. Anwar, et al. (2014); 2. Dahlberg y Aune (2013); 3. Ulfsdottir et al. (2018); 4. Nakano et al. (2012); 5. Aune y Dahlberg (2012); 6. Zand et al. (2017). 


\section{DISCUSIÓN}

A pesar de que cada artículo analizado presentaba objetivos diferentes y analizaba el empoderamiento desde una perspectiva distinta, se ha establecido una correlación entre ellos a través de la clasificación y categorización de los datos analizados.

En cuanto a la categoría «confianza depositada en otros», los estudios seleccionados tienen escasez de resultados, pero si se ha observado que en los artículos seleccionados de las revistas Birth, Women and Birth y Midwifery (Jöding et al., 2018; Lundqvist et al., 2002; Linfgren y Erlandson, 2010; Riggs et al., 2016) se habla de la importancia del establecimiento de una relación basada en la confianza entre la matrona y la paciente. Encontramos resultados similares en los estudios de Sánchez Jiménez et al. (2007) y de Mouta Oliveira (2017) cuando hablan de la importancia de la figura de la matrona en el proceso de empoderamiento, debido a su capacidad de fomentar la participación en la mejora de los autocuidados.

Si analizamos la categoría «sensación de control» en los artículos de las tres revistas estudiadas, se menciona el aumento de este sentimiento con una actividad simple como el sentirse escuchada por el profesional sanitario que la atiende (Jöding et al., 2018; Linfgren y Erlandson, 2010; Anwar et al., 2014). Esto se ve reforzado por Mouta Oliveira (2017), que afirma que a través del empoderamiento «las enfermeras pueden garantizar unas actuaciones basadas en el respeto, sin la utilización de intervenciones innecesarias reconociendo aspectos culturales y sociales que influyen en el proceso de parto», y por GobernaTricas y Jiménez-Segura (2013) cuando hacen referencia a la influencia de los aspectos sociales y culturales en el empoderamiento femenino.

Existen dos estudios de las revistas Birth y Midwifery (Linfgren y Erlandson, 2010; Aune y Dahlberg, 2012) en los que la categoría «aceptación» hace referencia a que el empoderamiento ayuda a las mujeres a aceptar que el parto es un proceso doloroso. Este aspecto ya se ha tenido en cuenta con el ofrecimiento del plan de parto a las madres gestantes, que ayuda a la embarazada a clarificar y dejar constancia de sus deseos, expectativas y necesidades durante el parto, facilitando la toma de decisiones informadas y el control de la situación (Biurrin-Garrido y Goberna Tricas, 2013). Sin embargo, en el artículo analizado de la revista Women and Birth, no se tiene en cuenta esta categoría. 
La comunicación como una capacidad a desarrollar, por parte de las profesionales, durante el proceso de empoderamiento a las mujeres (dentro de la categoría «capacidades») es un concepto que aparece en siete de los artículos seleccionados (Jöding et al., 2018; Ulfsdottir et al, 2018; Anwar et al., 2014; Riggs et al., 2016; Elien et al., 2017; Lundqvist et al., 2002). Este aspecto se hace indispensable cuando se trata de facilitar a las gestantes la toma de decisiones informadas y el desarrollo de la autonomía de la mujer (González Sanz et al., 2012).

En lo que a la categoría de «Soporte» se refiere, podemos distinguir entre soporte tácito y táctil. En el soporte tácito, se destaca la importancia de un apoyo físico, emocional y espiritual (Dahlberg y Aune, 2013; Nakano et al., 2012; Aune y Dahlberg, 2012, Linfgren y Erlandson, 2010), tanto por parte de la matrona como por parte de alguna persona cercana. Sin embargo, en el soporte táctil hay resultados combinados: algunos estudios resaltan la presencia física de la matrona como empoderadora (Anwar et al., 2014; Jördin et al., 2018); mientras que otros estudios defienden que el contacto físico en general de cualquier persona conocida (no la matrona específicamente) genera empoderamiento (Jördin et al., 2018; Linfgren y Erlandson, 2010).

En el estudio de González Sanz et al. (2012), se habla de la introducción de la humanización en el proceso de parto, por lo que se considera como una de las principales características del empoderamiento. En esta línea, cabe destacar que la humanización del parto en el proceso de empoderamiento femenino en el ámbito perinatal podría llevarse a cabo a través de los cuidados personalizados, como así lo defienden los artículos de Ulfsdottir et al., 2018; Zand et al., 2017; Anwar et al., 2014; Aunte y Dahlberg, 2012; la interacción entre la paciente y la matrona (Jördin et al., 2018; Leinfgren y Erlandsson, 2010) y la cercanía de esta última ( Dahlberg y Aune, 2013; Lundqvist et al., 2012), siendo la empatía otro aspecto a resaltar en los artículos de las revistas Midwifery y Birth (Lundqvist et al., 2002; Dalhberg y Aune, 2013).

Para finalizar, la categoría «Entorno» no se mantiene en las tres revistas, ya que solo Midwifery y Birth tienen artículos que hacen referencia a la necesidad de un espacio que induzca a la tranquilidad y al efecto de la presencia de personas cercanas. Por otro lado, la privacidad es considerada como un elemento clave en el proceso de empoderamiento femenino de las mujeres en el periodo perinatal (Riggs et al., 2016; Ulfsdottir et al., 2018). 
Las limitaciones para la realización de un estudio más amplio se encuentran en la necesidad de finalizar el estudio en un periodo de tiempo concreto, ya que este estudio ha sido realizado dentro del marco de un Trabajo fin de Máster del Máster en Investigación en Ciencias Sociosanitarias de la Universidad de León, disponiendo solo de algunos meses para la realización del mismo.

Junto a esta, que es la principal, hay otras limitaciones, como la dificultad de acceso a determinadas fuentes y el abordaje del empoderamiento femenino en el cuidado perinatal solo desde la esfera sanitaria, esto es, sin tener en cuenta otras publicaciones que pudieran tratarlo desde otras perspectivas como la social, la económica, la histórica, etc. Somos conscientes de la necesidad de tener en cuenta estas limitaciones en futuros trabajos sobre esta línea de investigación.

\section{CONCLUSIONES}

El empoderamiento femenino en el ámbito de la salud perinatal es un tema tratado escasamente en las revistas estudiadas. No obstante, su reparto entre las mismas es desigual, destacando la revista Birth como la que mayor atención presta a este tema.

En cuanto a la definición del concepto de empoderamiento se observa, en las revistas estudiadas, que no existe una definición unívoca del mismo, que permita una mejor integración de este término en el lenguaje enfermero.

En lo concerniente a los significados atribuidos a este término, destacan los siguientes: la confianza que se establece entre la matrona y las gestantes y sus familias; la receptividad por parte de la matrona respecto de las opiniones y preferencias expresadas por las gestantes; y la personalización de los cuidados, entendida como la atención basada en las necesidades concretas de cada mujer y no den planes de cuidados estandarizados.

Más allá de los objetivos planteados, hay que destacar que, aunque la literatura general indica que el empoderamiento femenino está relacionado directamente con la humanización del parto, son pocos los estudios, de entre los incluidos en este estudio, que se centran en esta relación.

Finalmente, considero que son precisas nuevas investigaciones que estudien el impacto del empoderamiento femenino durante el periodo perinatal sobre la salud de las madres y los recién nacidos/as, así como su calidad de vida posterior y en los cuidados que estos reciben durante la primera infancia. 


\section{BIBLIOGRAFÍA}

ANWAR, S. et al. Perinatal women's perceptions about midwifery led model of care in secondary care hospital in Karachi, Pakistan. En: Midwifery. 2014, vol. 30, pp. 7990. [Consulta: 22 de junio de 2018]. Disponible en: 〈doi.org/10.1016/j.midw.2013.10.020>.

Aune, I.; U. Dahlberg; O. I. Mag ART. Parents' experiences of midwifery students providing continuity of care. En: Midwifery. 2012, vol. 28, pp. 432-438. [Consulta:

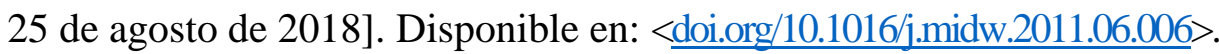

Biurrun-Garrido, A.; J. Goberna TriCAS. La humanización del trabajo de parto: necesidad de definir el concepto. Revisión bibliográfica. En: Matronas profesión. 2013, vol. 14, núm. 2, pp. 62-66. [Consulta: 10 de septiembre de 2018]. Disponible en:

<www.federacion-matronas.org/wp-content/uploads/2018/01/revisionbibliografica.pdf>.

Cardoso Da Silva Aires, C.M. et al. 2016. Empowerment in pregnancy: study on the adaptation of the empowerment scale for pregnant women to the portuguese context. En: Journal of nursing. 2016, vol. 4, núm. 10, pp. 49-56. [Consulta: 30 de octubre de 2018].

CAMberos SÁnchez, M.T. 2011. Empoderamiento femenino y políticas públicas, una perspectiva desde las representaciones sociales de género. En: Entramado. 2011, vol. 14, pp. 40-53. [Consulta: 22 de junio de 2018]. Disponible en: $\langle$ www.redalyc.org/articulo.oa?id=265422684003 >

CORAL IBARRA, R. Humanización del cuidado de enfermería durante el parto. En: U.D.C.A. Actualidad y Divulgación científica. 2008, vol. 11, núm. 1, pp. 47-56. [Consulta: 1 de julio de 2018]. Disponible en:

<www.scielo.org.co/scielo.php?script=sci_arttextypid=S012342262008000100007>.

DAhlberg, U.; I. Aune. The woman's birth experience: the effect of interpersonal relationships and continuity of care. En: Midwifery. 2013, vol. 29, pp. 407-415. [Consulta: 12 de agosto de 2018]. Disponible en: 〈www.index-f.com/para/n19/098.php〉.

Goberna Tricas, J.; M. C. Giménez Segura. Maternidad, Tecnología y Relación asistencial. En: Paraninfo digital. 2013, vol. 19. [Consulta: 27 de junio de 2018]. Disponible en: 〈doi.org/10.1016/j.midw.2012.09.006>.

KWEE, J.; H. MCBRIDE. Working together for women's empowerment: Strategies for interdisciplinary collaboration in perinatal care. En: Journal of Health Psychology. 
2015, vol. 1, núm. 2. [Consulta: 22 de julio de 2018]. Disponible en: <doi.org/10.1177/1359105315586211.hpq.sagepub.com>.

LINDGREN, H.; K. ERLANDSSON. Women's experiences of empowerment in a planned home birth: a swedish populaation-based study. En: Birth. 2010, vol. 37, núm. 4, pp. 309-317. [Consulta: 15 de septiembre de 2018]. Disponible en: <doi.org/10.1111/j.1523-536X.2010.00426.X >

LundQvist, A.; Nilstun, T; A. K. Dykes. Both empowered and powerless: Mothers' experiences of professional care when their newborn dies. En: Birth. 2002, vol. 29,

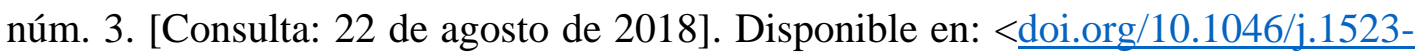
536X.2002.00187.x>.

LUQUE ORTEGA, Y. et al. Análisis bibliométrico de la producción científica sobre Gestion de casos. En: Paraninfo digital. 2010, vol. 10. [Consulta: 22 de junio de 2018]. Disponible en: 〈www.index-f.com/para/n10/p063.php〉

Mouta Oliveira, et al. Birth plan as a female empowerment strategy. En: Baiana enfermería. 2017, vol. 31, núm. 4. [Consulta: 1 de julio de 2018]. Disponible en: <www.portalseer.ufba.br/index.php./enfermagem/article/download/20275/15598>.

Perriman, N.; D. Lee Davis; S. Ferguson. What women value in the midwifery continuity of care model: a systematic review with meta-sythesis. En: Midwifery. 2018, vol. 62, pp. 220-229. [Consulta: 15 de septiembre de 2018]. Disponible en: <doi.org/10.1016/j.midw.2018.04.011>.

ReINA LEAL, L.M., et al. Producción y repercusión de revistas enfermeras en el Espacio Científico Iberoamericano 2012. En: Index de enfermería. 2013, vol. 22, núm. 4, pp. 259-266. [Consulta: 22 de julio de 2018]. Disponible en: < dx.doi.org/10.4321/S1132$12962013000300016>$.

RIGGS, E., et al. Cultural safety and belonging for refugee background women attending group pregnancy care: an autralian qualitative study. En: Birth. 2016, vol. 44, pp. 145-147. [Consulta: 1 de septiembre de 2018]. Disponible en: <doi.org/10.1111/birt.12272>.

SÁnChEZ-JiméneZ, B.; M. HernándeZ-Trejo; T. LARTIGUE-BECERRA. Estrategia educativa de enfermería para fortalecer el autocuidado durante el control prenatal. En: Perinatol Reprod Hum. 2007, vol. 21, pp. 167-177. [Consulta: 22 de julio de 2018]. Disponible en: <www.mediagraphic.com/cgi>. 
SimKIN, P. Just another day in a woman life? Women's long-term perceptions of their first birthexperience. En: Birth. 1991, vol. 18, núm. 4, pp. 203-210. [Consulta: 20 de junio de 2018]. Disponible en: 〈doi.org/10.1111/j.1523-536X.1991.tb00103.x〉.

SJÖDIN, M.; I. RADESTAD; S. ZWEDBERG. A qualitative study showing women's participation and empowerment in instrumental vaginal birth. En: Women and Birth. 2018, vol. 31, pp. 185-189. [Consulta: 2 de julio de 2018]. Disponible en: < $\underline{\text { doi.org/10.1016/j.wombi.2017.09.006> }}$.

SPANó NAKANO, A. M., et al. Childbirth experience according to a group of brazilian primiparas. En: Midwifery. 2012, vol. 28, pp. 844-849. [Consulta: 11 de agosto de 2018]. Disponible en: 〈doi.org/10.1016/j.midw.2011.09.014>.

ULFSDOTTIR, H., et al. Like a empowering micro-home: a quialitative estudy of women's. En: Midwifery. 2018, vol. 67, pp. 26-31. [Consulta: 27 de junio de 2018]. Disponible en: 〈www.doi.org/10.1016/j.midw.2018.09.004>.

YAM, E., et al. Introducing birth plans in Mexico: an exploratory study in a hospital serving low-income Mexicans. En: Birth. 2007, vol. 34, no. 1, pp. 42-48. [Consulta: 22 de julio de 2018]. Disponible en: 〈doi.org/10.1111/j.1523-536X.2006.00124.X >.

ZAND, D., et al. Parentingself-efficacy and empowerment among expectant mothers with substance use disorders. En: Midwifery. 2017, vol. 48, pp. 32-38. [Consulta: 15 de septiembre de 2018]. Disponible en: 〈doi.org/10.1016/j.midw.2017.03.003>. 